Marquette University

e-Publications@Marquette

Biomedical Engineering Faculty Research and Publications

Biomedical Engineering, Department of

2-14-2004

\title{
Changes in Pulmonary Arterial Wall Mechanical Properties and Lumenal Architecture with Induced Vascular Remodeling
}

Robert C. Molthen

Marquette University, robert.molthen@marquette.edu

Amy Heinrich

Medical College of Wisconsin

Steven Thomas Haworth

Medical College of Wisconsin

Christopher A. Dawson

Medical College of Wisconsin

Follow this and additional works at: https://epublications.marquette.edu/bioengin_fac

Part of the Biomedical Engineering and Bioengineering Commons

\section{Recommended Citation}

Molthen, Robert C.; Heinrich, Amy; Haworth, Steven Thomas; and Dawson, Christopher A., "Changes in Pulmonary Arterial Wall Mechanical Properties and Lumenal Architecture with Induced Vascular Remodeling" (2004). Biomedical Engineering Faculty Research and Publications. 123.

https://epublications.marquette.edu/bioengin_fac/123 


\title{
Changes in pulmonary arterial wall mechanical properties and lumenal architecture with induced vascular remodeling
}

\author{
Robert C. Molthen ${ }^{\mathrm{a}, \mathrm{b}, \mathrm{c}}$, Amy E. Heinrich ${ }^{\mathrm{a},}$, Steve T. Haworth ${ }^{\mathrm{a}}$, and Christopher A. Dawson ${ }^{\mathrm{a}, \mathrm{b}, \mathrm{c}}$ \\ ${ }^{a}$ Department of Medicine, Medical College of Wisconsin, Milwaukee, WI, 53226 \\ ${ }^{\mathrm{b}}$ Department of Biomedical Engineering, Marquette University, Milwaukee, WI 53203 \\ ${ }^{\mathrm{c}}$ Zablocki VA Medical Center, Milwaukee, WI, 53295
}

\begin{abstract}
To explore and quantify pulmonary arterial remodeling we used various methods including micro-CT, high-resolution 3dimensional x-ray imaging, to examine the structure and function of intact pulmonary vessels in isolated rat lungs. The rat is commonly used as an animal model for studies of pulmonary hypertension $(\mathrm{PH})$ and the accompanying vascular remodeling, where vascular remodeling has been defined primarily by changes in the vessel wall composition in response to hypertension inducing stimuli such as chronic hypoxic exposure (CHE) or monocrotaline (MCT) injection. Little information has been provided as to how such changes affect the vessel wall mechanical properties or the lumenal architecture of the pulmonary arterial system that actually account for the hemodynamic consequences of the remodeling. In addition, although the link between primary forms of pulmonary hypertension and inherited genetics is well established, the role that genetic coding plays in hemodynamics and vascular remodeling is not. Therefore, we are utilizing Fawn-Hooded (FH), Sprague-Dawley (SD) and Brown Norway (BN) rat strains along with unique imaging methods to parameterize both vessel distensibility and lumenal morphometry using a principal pulmonary arterial pathway analysis based on self-consistency. We have found for the hypoxia model, in addition to decreased body weight, increased hematocrit, increased right ventricular hypertrophy, the distensibility of the pulmonary arteries is shown to decrease significantly in the presence of remodeling.
\end{abstract}

Keywords: Micro-CT, vascular remodeling, pulmonary artery, mechanical properties, lumenal architecture

\section{INTRODUCTION}

The rat is a commonly used model for PH. The vascular remodeling that occurs with the onset and progression of $\mathrm{PH}$ has been the focus many studies that addressed the histological changes seen in the pulmonary arteries (PA) such as extension of smooth muscle into small, previously non-muscular arteries, medial thickening in normally muscular arteries, and a decrease in the number of arteries that filled with a barium containing casting material injected at high pressure ${ }^{1,2,3,4}$. There is evidence of differences in the architecture of the remodeling seen in the CHE and MCT models ${ }^{5}$ as well as differences between therapeutic interventions in the two models ${ }^{6}$. In addition, the literature also describes differences in hemodynamic and vascular traits between rat strains $7,8,9$. The FH rat has a genetic susceptibility to pulmonary hypertension 7.8 . To understand the genetic component, it becomes important to study pulmonary vascular remodeling and how it differs in various strains of rats a with different disease models. A number of groups have investigated differences between the CHE and MCT models 5, 6, 10 using hemodynamic and histological methods. However, the intact structure of the pulmonary arterial tree is not retained and not describable through histological evaluation and although changes in the relative constitution of the vessel walls are undeniably important, the arterial tree geometry and the vessel wall mechanical properties determine the pulmonary arterial function. Importantly, there remains little information available as to how these properties are affected by the remodeling process. This work investigates identifying differences in the morphometry and the mechanical properties of the intact pulmonary arterial trees of the SD, FH and SD rats in both the CHE and MCT model.

In order to define their physiological properties, techniques are needed to describe the branching structure represented by the pulmonary arterial tree. There is a large amount of data analysis and tabulation required to fully describe the intact arterial tree. Complete documentation of even a single arterial tree would not only require enormous effort, but important characteristics of structure/function relationships can be lost in the attempt to analyze and quantify such a large amount of information. Previously investigators have studied methods of representing airway and arterial 
tree structures ${ }^{11,12}$. Various approaches have been investigated to summarizing complex branching networks ${ }^{12,13,14,15}$. We have adopted the concept of self-consistency ${ }^{16,17}$ within the pulmonary arterial tree and exploits its recursive structural properties to reduce the measurements to only the main trunk and the inlet to its daughter branches ${ }^{18}$. In this manner, a self-consistent tree can be defined as one in which all subunits (sub-trees) downstream from any segment with a given diameter are similar, at least in a statistical sense and the data set still holds requisite hemodynamic information for analysis of arterial flow. Using this reduction technique we have developed various models to describe the principal pathway, the main pulmonary arterial trunk and its daughter branches.

\section{METHODOLOGY}

\subsection{Rat Preparations}

$\mathrm{SD}, \mathrm{FH}$ and $\mathrm{BN}$ rats approximately 65 days of age, ranging in weight from $250-350$ gm were used for this study. Rats were challenged by induction of pulmonary hypertension or untreated and used as controls. PH was induced through either exposure to hypoxia, in an isobaric chamber maintained at $10 \%$ oxygen, or with a single injection of monocrotaline (60 mg/kg sub-Q). The hypoxic group was subject to CHE for 21 days, the MCT group lived under local standard animal care procedures for 28 days. Control rats were kept in a normoxic environment ( $21 \%$ oxygen) for 21 days also under standard care. All groups were allowed to eat and drink ad libitum. At the time of sacrifice each rat was anesthetized with sodium pentobarbital $(50 \mathrm{mg} / \mathrm{kg} \mathrm{IP})$ and a midline sternotomy performed. The rat was then heparinzed (200 IU/kg) by right ventricular injection and then exanguinated with samples of the removed blood centrifuged fro analysis of hematocrit. The trachea and PA were cannulated (PE 240 tubing) and the lungs removed. The lungs were hung by the two cannulae in a fixture designed for both hemodynamic and imaging studies. The tracheal cannula was connected to a ventilator and the arterial cannula to a temperature controlled perfusion circuit that allowed constant monitoring of the PA pressure. The lungs were constantly ventilated with a $15 \% \mathrm{O}_{2}, 6 \% \mathrm{CO}_{2}$ in $\mathrm{N}_{2}$ gas mixture, 3 mmHg end expiratory pressure and $8 \mathrm{mmHg}$ end inspiratory pressure. Serveral breaths with peak inspiratory pressures of $15-20$ $\mathrm{mmHg}$ were performed to eliminate any atelectasis that might have occurred during the excision and help clear blood from the vessels. The lungs were perfused with a physiological salt solution (pss) containing 5\% serum albumin. Hemodynamic perfusion studies were performed at flows rates of 5,10,20,30 and $40 \mathrm{ml} / \mathrm{min}$ before and after $6 \mathrm{mg}$ of papaverine hydrochloride was added to the reservoir of perfusate. After the hemodynamic data was recorded, the cannulae were clamped at end inspiration and the lungs transported to an enclosed imaging chamber within the imaging suite.

\subsection{Imaging Methods}

The pss in the arterial tree was then replaced by filling it with perfluorooctyl bromide (perflubron) for intravascular x-ray absorption. Tracheal pressure was set to $6 \mathrm{mmHg}$, and the pleura exposed to the atmosphere. The lungs were rotated in the $\mathrm{x}$-ray beam to obtain 360 planar images at intravascular pressures of 5.4, 12, 21 and $30 \mathrm{mmHg}$ using a height adjustable reservoir. At these pressures, Perflubron tends not pass through the capillaries, therefore only the arteries fill with contrast medium. The CT scanning at each pressure takes approximately 4 minutes and several minutes were allowed between scans for the vessels to reach equilibrium after a change in intravascular pressure was made. Threedimensional Feldkamp ${ }^{19}$ cone-beam reconstruction was performed on the projection data. Detailed measurements were made on the resultant CT data sets acquired from the pulmonary arterial trees, using a principal pulmonary arterial pathway analysis model based on self-consistency ${ }^{18}$. Data on the changes in morphology and passive mechanical properties of the arteries were measured.

\subsection{Imaging System and Reconstruction}

The micro-focal x-ray CT system is composed of a Feinfocus FXE/FXT 100.20 microfocal x-ray source (3um effective focal spot), a Thomson TH9438 HX H661 VR24 image intensifier (II) optically coupled to a Silicon Mountain Design (SMD1M-15) CCD, and a New England Affiliated Technologies (NEAT) specimen micromanipulator stage, all mounted on a precision rail with positional information provided by Mitutoyo linear encoders accurate to $10 \mu \mathrm{m}$. The geometry of the imaging system allows magnification of the specimen to be increased by 1) decreasing the specimen's proximity to the $x$-ray source or 2) increasing the distance of the II from the source. Each planar projection image was a result of averaging 7 frames to minimize noise. The image data (512x512 pixels) was sent from the camera, via RS-422, to a frame grabber board mounted in a Dell 610 workstation running WindowsNT. Image acquisition and positional control were all preformed by window-based software, written in-house, running on the 610 workstation. The projection data was transferred via network to a Dell 340 workstation running Linux Red Hat and after proper preprocessing, that 
compensates for field distortions and nonuniformities introduced by the imaging chain, isotropic reconstructions were obtained through an implementation of the Feldkamp cone-beam algorithm ${ }^{19}$. The typical reconstructed volume was $497^{3}$ pixels with a typical pixel size of approximately $70 \mu \mathrm{m}$.

\subsection{Measuring Arterial Lengths and Diameters}

Measurement of parameters available through CT imaging of contrast filled vessels include the lumenal diameter of the artery as well as the arterial trunk length. Morphometric measurements were made, in the principal pathway of the pulmonary arterial trunk, from the reconstructed data sets using methods introduced in earlier work ${ }^{18,20,21}$. However, since these have been published, new Matlab algorithms developed allow manipulation of isotropic sub-volumes to insure accurate placement of the 2-D measurement plane, see figure 5. Diameter measurements were made midway between successive bifurcations along the main pulmonary trunk and all attached daughter branches, while data on vessel length was obtained from bifurcation-to-bifurcation positional information. Lumenal measurements were made in arteries ranging in size from approximately $70-2500 \mathrm{um}$. Since information on vessel wall mechanical properties is fundamental to this study, to measure mechanical properties of the vessel wall, measurements of the each lung at several different intravascular or transpulmonary pressures were made at identical locations on the principal pathway.

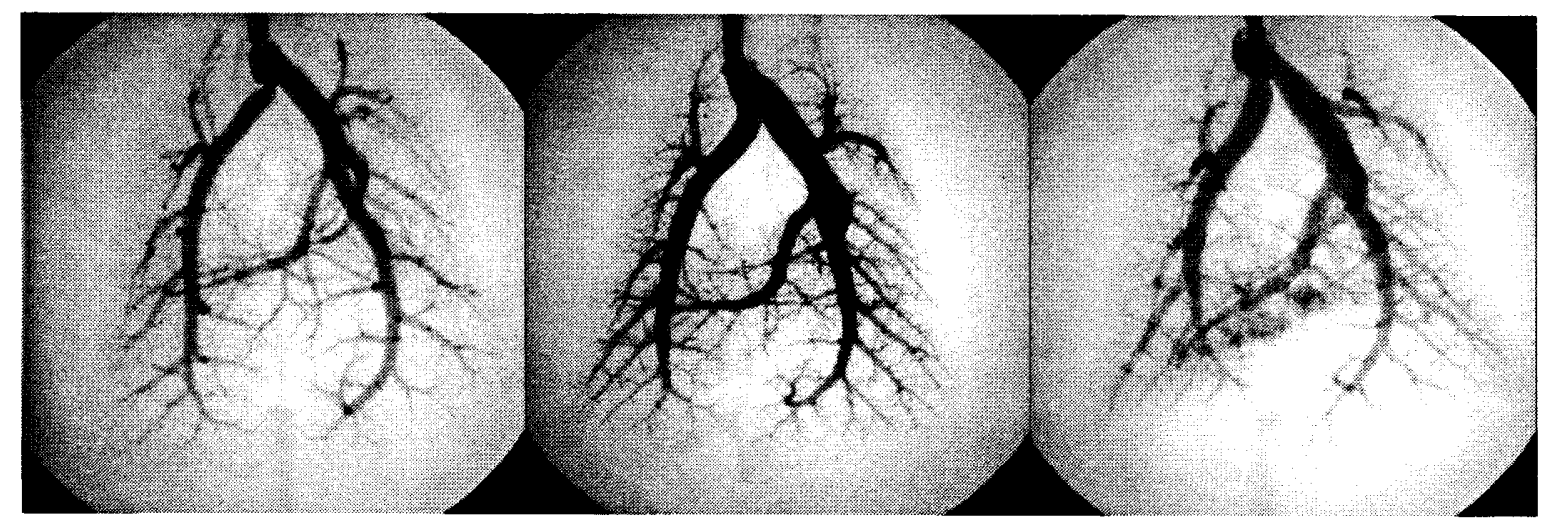

Figure 1. Planar pulmonary arteriograms of a Fawn-Hooded, Sprague-Dawley, and Brown-Norway (respectively, left to right). The arteries are filled with perflubron for $\mathrm{x}$-ray contrast. The intravasuclar pressure $30 \mathrm{mmHg}$ and transpulmonary pressures of $6 \mathrm{mmHg}$.

\section{RESULTS}

\subsection{Planar radiographic images}

With visual inspection, simple planar angiographic images can provide useful qualitative representation of the differences seen in the three rat strains used in this study. Presented in figure 1 are pulmonary arteriograms from a FH, $\mathrm{SD}$, and $\mathrm{BN}$ rat lung. The pulmonary arterial trees from the three rat strains have a similar gross appearance, however the arteries in the Sqrague-Dawley and BN rats exhibit slightly more distension for the same intravascular pressure of 30 $\mathrm{mmHg}$. The BN lung has leakage of the contrast agent near the perialveolar region in the cardiac lobe, an event found to be typical of this strain. The FH lung shows signs of vessel drop out or a lack of filling in the small arteries and arterioles, as evident from the lack of background haze in the region of the parenchyma.

By examining the arteriograms, differences in the vessel distensibility are also apparent between the rat strains. Figures 2 and 3 give examples of these differences. The FH left lungs shown in figure 2 reveal the difference in arterial tree morphometry at $30 \mathrm{mmHg}$ compared to $5 \mathrm{mmHg}$ in the normoxic control, top row. A change in main trunk vessel diameter is evident with the change in intravascular pressure. In the bottom row in figure 2 there are planar images of left lungs from a hypoxia exposed FH rat. Again, differences in the trunk diameters are seen between the high and low pressure images, however, in the hypoxic lung compared to the control lung the diameter change is smaller and the lack of arterial filling is much more evident. Similar trends are seen in figure 3, in which planar projections of the left lobe of two BN lungs are presented (normoxic on the top row and hypoxic on the bottom row). In this panel, there is a large change in the trunk diameters seen in the normoxic lung between high and low intravascular pressures. When comparing the FH lungs to the BN lungs, the vessels appear more distended in the BN lungs at each pressure and the hypoxic BN lungs lung appears to have less remodeling. 


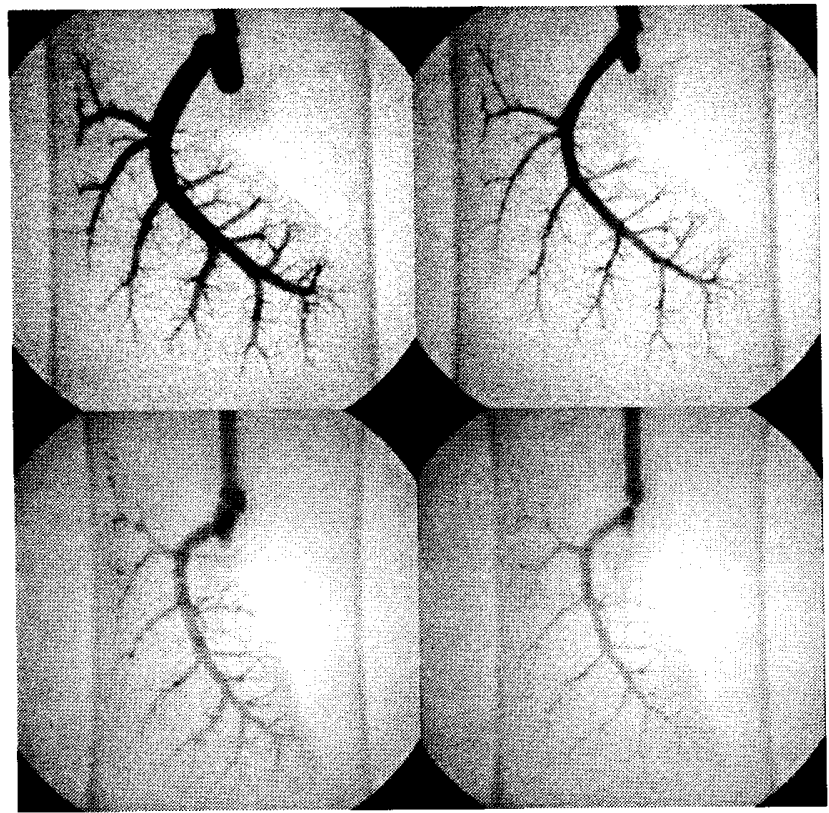

Figure 2. (Top row) Planar pulmonary arteriograms of the left lung from a normoxic (control) Fawn-Hooded rat. The arteries are filled with perflubron at an intravascular pressure $30 \mathrm{mmHg}$ (left) and $5 \mathrm{mmHg}$ (right) and transpulmonary pressures of $6 \mathrm{mmHg}$. (Bottom row) Planar pulmonary arteriograms of the left lung from a hypoxic Fawn-Hooded rat. The intravascular pressure $30 \mathrm{mmHg}$ (left) and $5 \mathrm{mmHg}$ (right) and transpulmonary pressures of $6 \mathrm{mmHg}$. The cannula at the top of each lung is $1.67 \mathrm{~mm}$ in diameter.

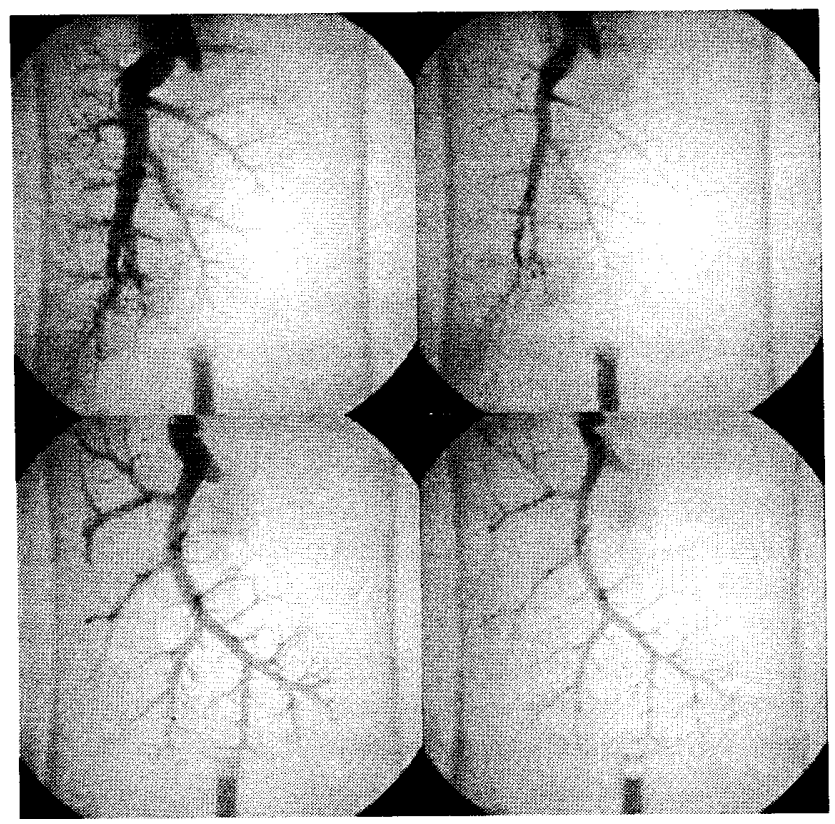

Figure 3. (Top row) Planar pulmonary arteriograms of the left lung from a normoxic (control) Brown-Norway rat. The arteries are filled with perflubron at an intravascular pressure $30 \mathrm{mmHg}$ (left) and $5 \mathrm{mmHg}$ (right) and transpulmonary pressures of $6 \mathrm{mmHg}$. (Bottom row) Planar pulmonary arteriograms of the left lung from a hypoxic Brown-Norway rat. The intravascular pressure $30 \mathrm{mmHg}$ (left) and $5 \mathrm{mmHg}$ (right) and transpulmonary pressures of $6 \mathrm{mmHg}$. The cannula at the bottom of each lung is $1.67 \mathrm{~mm}$ in diameter. 


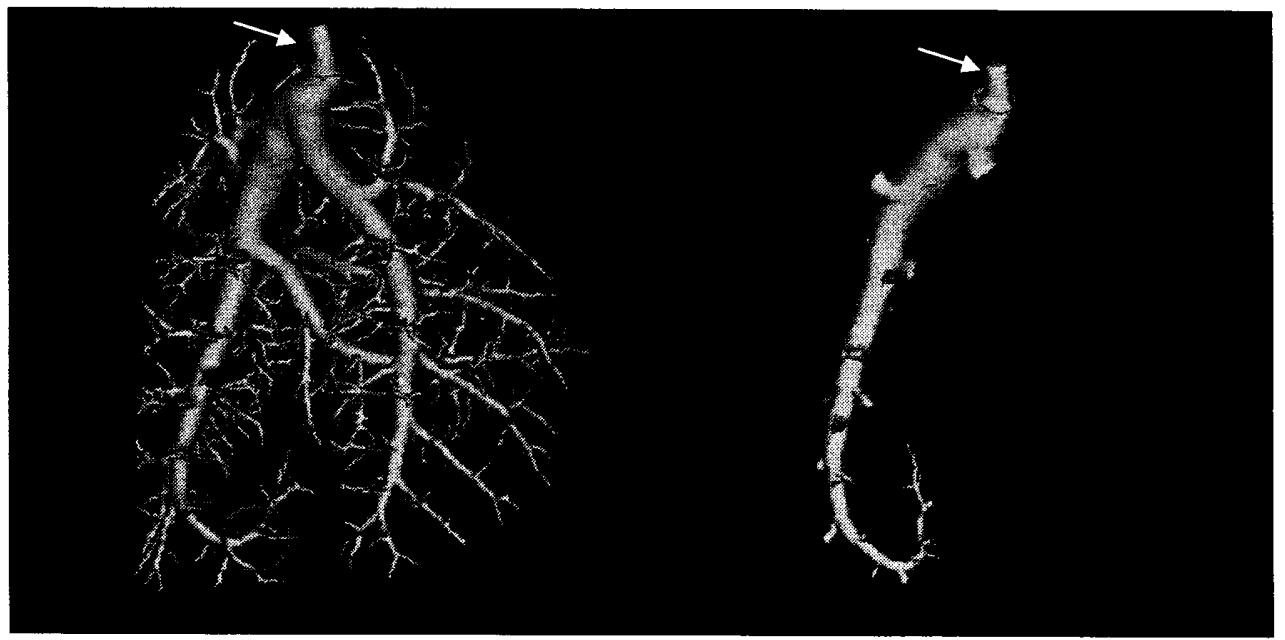

Figure 4. A thresholded surface shaded rendering ${ }^{22,23}$ of a normoxic Sprague-Dawley rat lung (left). The red circle is drawn to denote an example of the region of the arterial tree being measured in figure 5. On the left is a rotated surface shaded rendering of the principal pathway from the same arterial tree. The arrows points to the PE 240 cannula, it has an inner diameter of $1.76 \mathrm{~mm}$.

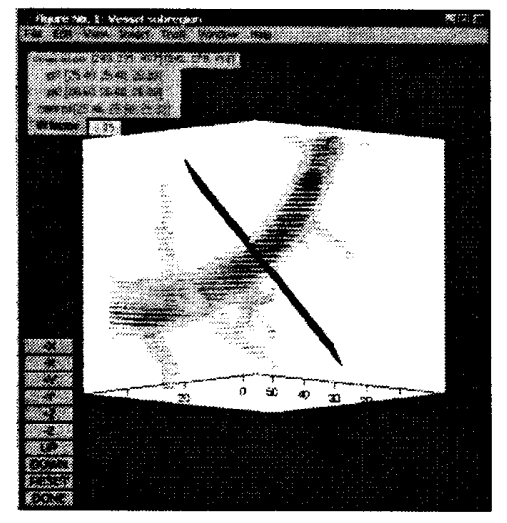

Figure 5. A screen capture of the user interface which allows a precise manipulation of the measurement plane (in black) in order to assure that it is perpendicular to the vessel's central axis (vessel in pseudo color seen on white 3D coordinate space background) that gives the best representation of the vessel's cross-section and provides accurate diameter estimation.

\subsection{Distensibility measurements}

In order to measure vessel distensibility, the pulmonary arterial tree of each lung was imaged, reconstructed and the principal pathway measured at 4 intravascular pressures, similar to the procedures carried out in previous work ${ }^{20,21}$. An example of an isotropic CT data set of a rat pulmonary arterial tree and its principal pathway at an intravascular pressure off $30 \mathrm{mmHg}$ are presented in figure 4. Isotropic reconstruction allows infinite positioning of the plane that will contain data from which the diameter estimations are made. This plane is manually manipulated until it is perpendicular to the vessel's central axis to provide the most accurate diameter estimation as in figure 5. To calculate each individual 
vessel's distensibility, a linear regression of diameter vs. pressure is performed. For the pressure range used to run the experiments, the diameter versus pressure relationship is fairly linear (7). This regression gives a slope $(\beta)$, the distensibility, and an intercept (D0), an estimate of the undistended diameter of the vessel segment. Furthermore, we have found the relationship between $\beta$ and D0 for each lung can be modeled linearly with an intercept that does not differ significantly from zero. The plot of $\beta$ vs. D0 for an example SD rat lung is presented in figure 6 . This implies the distensibility of all the vessel within the lung are diameter independent and that this distensibility can be described with one parameter, the slope of the $\beta$ vs. D0 relation or $\alpha$. Values of $\alpha$ are presented in figure 7a for the various rat strains with and without chronic hypoxic exposure. The hypoxic exposure causes the distensibility of the arteries to decrease. The largest change in distensibility is seen in the $\mathrm{FH}$ rat. In figure $7 \mathrm{~b}$ the pulmonary arterial distensibility for the chronic hypoxic, and monocrotaline treated SD rat are given. Distensibility also significantly decreases in the monocrotaline model. At 28 days the remodeling in the MCT rats appears to be equal to or slightly less than the hypoxic model days.

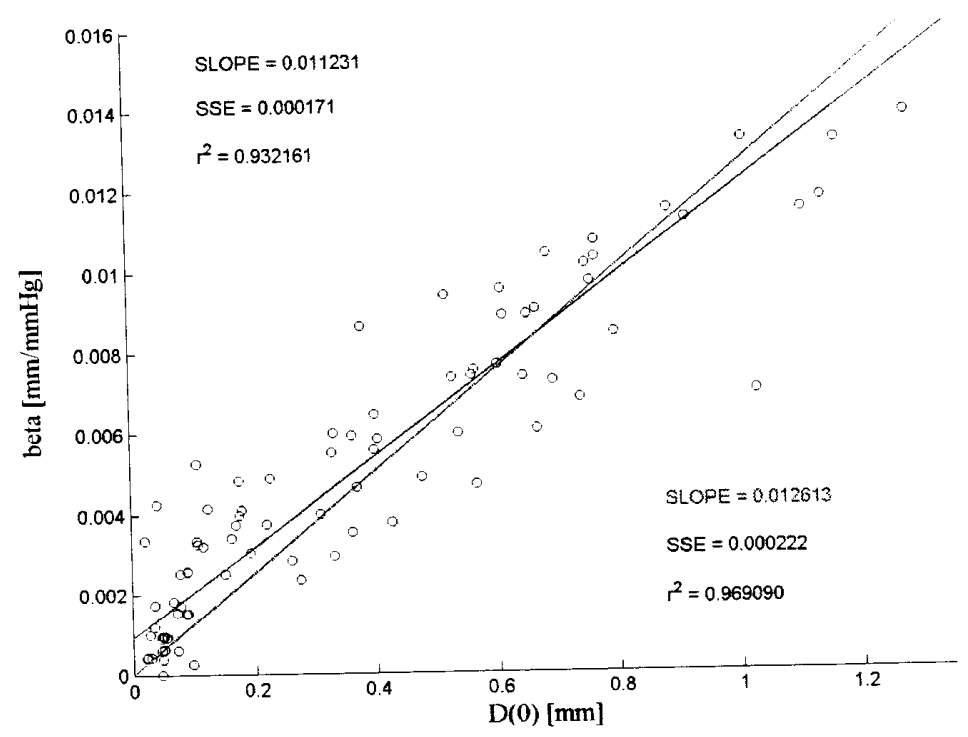

Figure 6. $\beta$ vs. D0 plotted for an example Sprague-Dawley lung. The actual regression line is compared to the model forced through zero. The slope of the model is equal to $\alpha$.

\subsection{Isolated lung hemodynamics}

In addition to information about the vessel distensibility, hemodynamic data that reflects the response of the lung vasculature to changes in flow is an important benchmark for understanding pulmonary vascular resistance and the load seen by the right heart. The results of perfusion studies performed in the isolated lungs are presented in figure 8 . Chronic Hypoxic exposure causes an increase in the perfusion pressure for each strain of rat. The BN and SD rats have similar baseline (control) and post-hypoxia pressures. The FH rat has a slightly elevated baseline and a much larger increase in pulmonary vascular resistance after hypoxic exposure. The pressure vs. flow in SD and FH rats before and after monocrotaline treatment is plotted in figure 9. Although the baseline and post-treatment pressure are higher in the FH rat, both strains appear to have an equal increase perfusion pressure.distensibility, a linear regression of diameter vs. pressure is performed. For the pressure range used to run the experiments, the diameter versus pressure relationship is fairly linear ${ }^{24}$. This regression gives a slope $(\beta)$, the distensibility, and an intercept $\left(D_{0}\right)$, an estimate of the undistended diameter of the vessel segment. Furthermore, we have found the relationship between $\beta$ and $D_{0}$ for each lung can be modeled linearly and has an intercept that does not differ significantly from zero. The plot of $b$ vs. D0 for an example SD rat lung is plotted in figure 6 . This implies that the distensibility of all the vessel within the lung are diameter independent and that this distensibility can be described by one parameter, the slope of the $\beta$ vs. $D_{0}$ relation $(\alpha)$. Values 


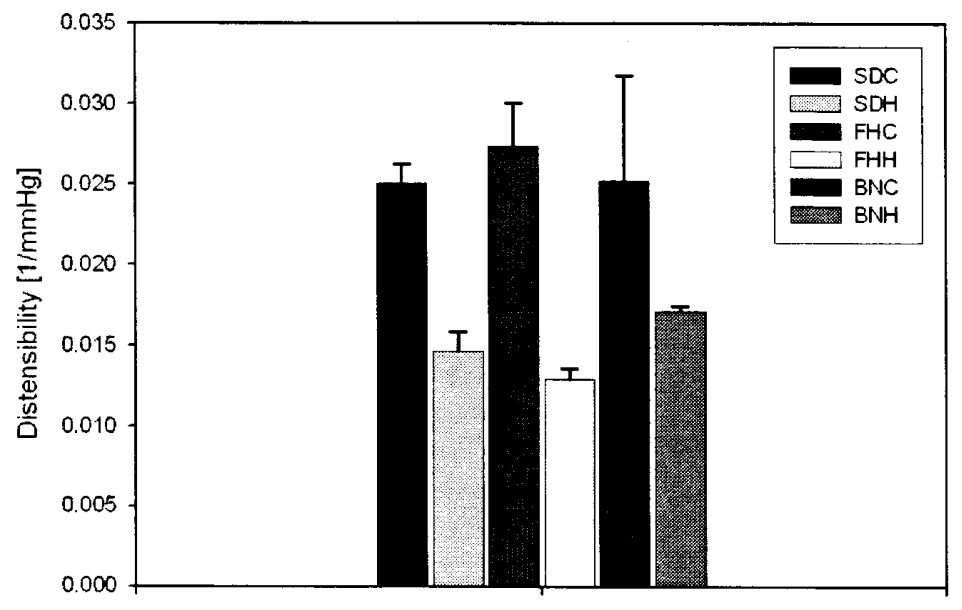

Experimental group

Figure 7a. Pulmonary arterial distensibility (mean \pm SE) in Sprague-Dawley control (SDC, $N=6$ ), Sprague-Dawley hypoxic ( $\mathrm{SDH}, \mathrm{N}=6$ ), Fawn-Hooded control (FHC, N=6), Fawn-Hooded hypoxic (FHH, N=6), Brown-Norway control (BNC, N=2), and Brown-Norway hypoxic (BNH, $\mathrm{N}=2$ ) rats.

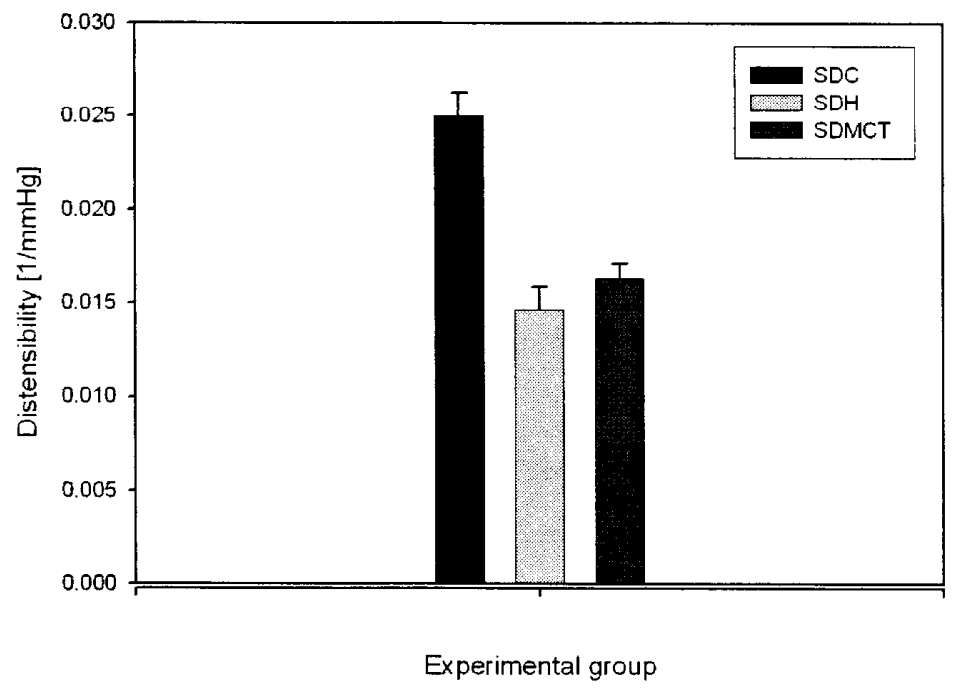

Figure 7b. Pulmonary arterial distensibility (mean \pm SE) in Sprague-Dawley control (SDC, $\mathrm{N}=6$ ), hypoxic ( $\mathrm{SDH}, \mathrm{N}=6$ ), and monocrotaline treated (SDMCT, $\mathrm{N}=6$ ) rats.

of $\alpha$ are presented in figure 7a for the various rat strains with and without chronic hypoxic exposure. The hypoxic exposure causes the distensibility of the arteries to decrease. The largest change in distensibility is seen in the FH rat. In figure $7 \mathrm{~b}$ the pulmonary arterial distensibility for the control, chronic hypoxic, and monocrotaline treated SD rat. Distensibility also significantly decreases in the monocrotaline model. At 28 days the remodeling in the MCT rats appears to be equal to or slightly less than the hypoxic model at 21 days. 


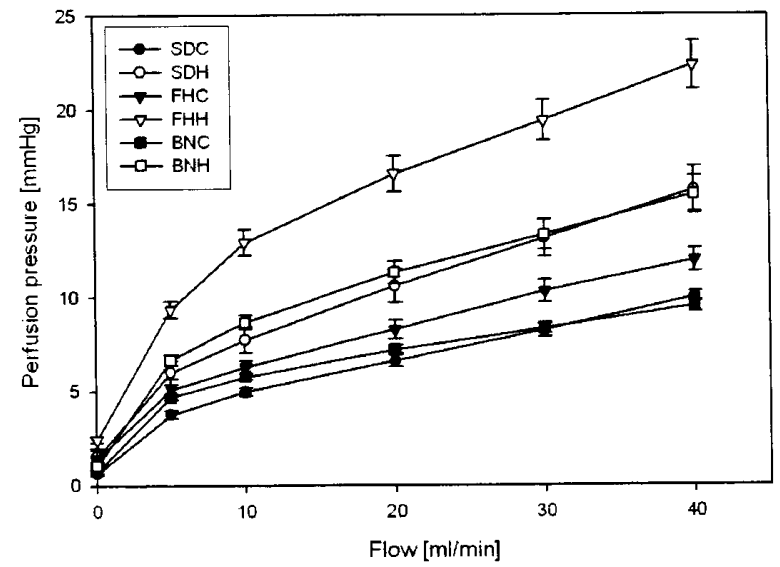

Figure 8. Perfusion pressures (mean $\pm \mathrm{SE}$ ) in Sprague-Dawley control (SDC, $\mathrm{N}=6$ ), Sprague-Dawley hypoxic (SDH, N=6), Fawn-Hooded control (FHC, N=4), FawnHooded hypoxic ( $\mathrm{FHH}, \mathrm{N}=7$ ), Brown-Norway control ( $\mathrm{BNC}, \mathrm{N}=7$ ), and BrownNorway hypoxic $(\mathrm{BNH}, \mathrm{N}=7)$ rats.

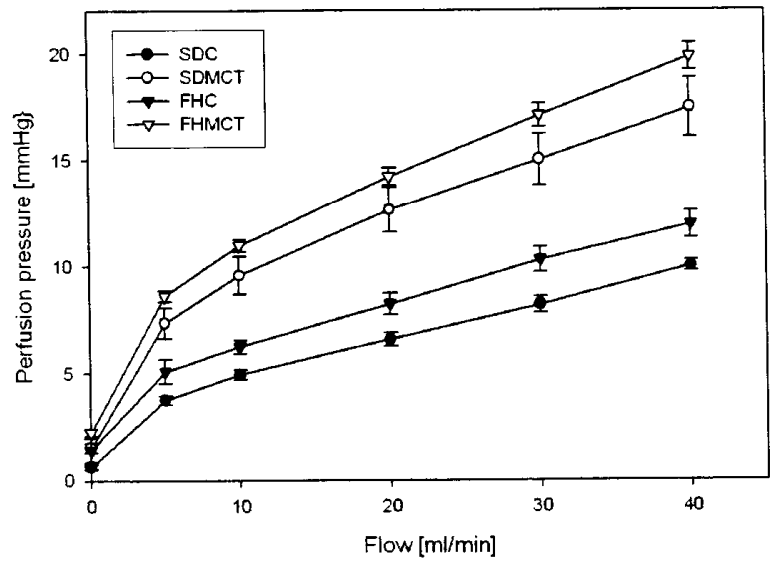

Figure 9. Perfusion pressures (mean $\pm S E$ ) in Sprague-Dawley control (SDC, $N=6$ ), Sprague-Dawley monocrotaline treated (SDMCT, N=6), Fawn-Hooded control (FHC, N=4), Fawn-Hooded monocrotaline treated (FHMCT, N=6) rats.

\subsection{A phenotypic vector}

To begin to compare hemodynamic data with arterial distensibility and examine the impact of morphological differences between the groups, we have described a set (or morphology vector) of quantitative parameters that can be measured. A description of the models use in this work can be found in work by Karau et. al. ${ }^{18}$. It describes models of the main trunk and daughter branch diameter as a function of distance along the trunk with respect to the inlet (first lobar bifurcation). Parameters from these models that are part of this quantitative vector include: $\mathrm{D}_{0}$, the estimated diameter of the main trunk at its inlet, $\mathrm{D}_{\mathrm{Br}}$, the estimated diameter of the first daughter branch extrapolated back to the inlet of the main trunk, $\mathrm{L}_{\mathrm{to}}$, the total length of the principal pathway, $\mathrm{N}_{\mathrm{tot}}$, an approximation of the total number of branches off the main trunk, $c$, the concavity or convexity of the taper of the main trunk, $b$ a measure of how the branches are distributed along the main trunk, $\mathrm{CV}_{\mathrm{Br}}$, the coefficient of variation obtained from the variance between measurements made of the daughter 
branch diameters and the model, $\mathrm{D}_{0} / \mathrm{L}_{\mathrm{tot}}$, a ratio of two of the parameters that accounts for variations in scale. The values of this parameter vector are given for the three rat strains in table 1. $\alpha$ or the arterial distensibility is strong component of the morphology vector. The other parameters seem to be less correlated to hypoxic exposure. However there is a trend for some of the vector components of the SD and BN strains that both decrease while there is and opposite trend in the FH rat. The value of $c$, that reflects the curvature of the taper of the main trunk appears to decrease with hypoxic exposure and more so in the SD and BN strains.

\begin{tabular}{|c|c|c|c|c|c|c|c|c|c|c|c|c|}
\cline { 2 - 12 } \multicolumn{1}{c|}{} & \multicolumn{2}{c|}{ SDC } & \multicolumn{2}{c|}{ SDH } & \multicolumn{2}{c|}{ FHC } & \multicolumn{2}{c|}{ FHH } & \multicolumn{2}{c|}{ BNC } & \multicolumn{2}{c|}{ BNH } \\
\cline { 2 - 12 } \multicolumn{1}{c|}{} & \multicolumn{2}{c|}{$\mathrm{N}=6$} & \multicolumn{2}{c|}{$\mathrm{N}=6$} & \multicolumn{2}{c|}{$\mathrm{N}=6$} & \multicolumn{2}{c|}{$\mathrm{N}=6$} & \multicolumn{2}{c|}{$\mathrm{N}=2$} & \multicolumn{2}{c|}{$\mathrm{N}=2$} \\
\cline { 2 - 12 } \multicolumn{1}{c|}{} & MEAN & SE & MEAN & SE & AVR & SE & AVR & SE & AVR & SE & AVR & SE \\
\hline D(0) & 1.5276 & 0.0333 & 1.4291 & 0.0442 & 1.3233 & 0.1651 & 1.3733 & 0.2333 & 1.3928 & 0.0510 & 1.3505 & 0.0214 \\
DBr $_{\text {B }}$ & 0.6055 & 0.0336 & 0.4967 & 0.0189 & 0.5255 & 0.0775 & 0.5290 & 0.0531 & 0.5967 & 0.0107 & 0.5540 & 0.0360 \\
Ltot & 38.1779 & 2.3579 & 38.7820 & 0.9966 & 39.5333 & 3.1992 & 38.5167 & 3.5963 & 33.3906 & 5.2710 & 39.2275 & 1.6475 \\
Ntot & 32.3710 & 1.1669 & 31.6507 & 2.1109 & 33.1429 & 1.5496 & 33.0667 & 1.0667 & 30.3806 & 3.0758 & 30.5275 & 2.8353 \\
C & 0.9097 & 0.0397 & 0.8496 & 0.0659 & 0.7917 & 0.0677 & 0.7653 & 0.0816 & 0.8713 & 0.0067 & 0.7884 & 0.0004 \\
b & 1.9320 & 0.1223 & 1.6427 & 0.2031 & 1.6200 & 0.0747 & 1.7747 & 0.1270 & 1.9858 & 0.1242 & 1.6784 & 0.1635 \\
$\alpha$ & 0.0250 & 0.0012 & 0.0147 & 0.0012 & 0.0274 & 0.0026 & 0.0129 & 0.0007 & 0.0252 & 0.0065 & 0.0171 & 0.0003 \\
CV_br & 0.5385 & 0.0290 & 0.5690 & 0.0449 & 0.5928 & 0.1360 & 0.6071 & 0.0683 & 0.4798 & 0.0036 & 0.4706 & 0.0234 \\
D(0)/Ltot & 0.0387 & 0.0033 & 0.0365 & 0.0017 & 0.0338 & 0.0068 & 0.0356 & 0.0046 & 0.0430 & 0.0083 & 0.0345 & 0.0020 \\
\hline
\end{tabular}

Table 1. Morphometric parameters from Sprague-Dawley control (SDC, N=6), Sprague-Dawley hypoxic (SDH, N=6), Fawn-Hooded control (FHC, N=6), Fawn-Hooded hypoxic (FHH, N=6), Brown-Norway control (BNC, N=1), and Brown-Norway hypoxic $(\mathrm{BNH}, \mathrm{N}=2)$ rats.

\section{DISCUSSION}

A feature in the pulmonary arterial tree that significantly changes with either hypoxic exposure or monocrotaline treatment is distensibility of the vessels. All rat strains studied show decreased pulmonary arterial distensibility after chronic hypoxic exposure. The arterial distensibility of the FH rat in response to hypoxia appears to decrease in larger proportion than the SD or BN rat. However, since the number of BN CT data sets that have been analyzed up to this point are relatively small, the strength of this conclusion needs to be validated. Therefore, distensibility and morphology vector data values for the BN strain are preliminary at this time. The fact that perfusion studies in the $\mathrm{FH}$ animals at baseline do not correlate well with the distensibility data is interesting. It would suggest that FH arteries may have slightly more distensible vessels at baseline, but have higher baseline perfusion pressures. This would suggest mechanisms other than vessel distensibility are causing an increase in FH perfusion pressures under normoxic conditions. It will also be interested to find that a similar trend for vessel distensibility holds true for FH monocrotaline challenge since the increase in perfusion pressures is similar for the SD and FH monocrotaline treated rats. It appears that the differences in pulmonary vascular remodeling are subtle but important. Ultrastructural differences have been seen between the hypoxic and monocrrotaline models, with immature smooth muscle cells (SMCs) and indistinct elastic laminae present in the monocrotaline model compared to mature SMCs and dense laminae in hypoxia model ${ }^{25}$. Bioactive NO may also play an important role in monocroaline induced $\mathrm{PH}^{26}$. To elucidate some of these characteristic differences, it is hoped that the subtle but parallel shifts seen between the SD and BN strains have a measurable effect on the morphology vector, vessel distensibility and that the effects can be determined after $\mathrm{N}$ has been increased.

\section{ACKNOWLEDGEMENTS}

This work supported by HL 19298, HL64368, the W.M. Keck Foundation and the Department of Veterans Affairs. 


\section{REFERENCES}

1. M. Rabinovitch, M. A. Konstam, W. J. Gamble, N. Papanicolaou, M. J. Aronovitz, S. Treves, and L. Reid, "Changes in pulmonary blood flow affect vascular response to chronic hypoxia in rats," Circulation research, 52, pp. 432-441, 1983.

2. N. F. Voelkel and R. M. Tuder, "Hypoxia-induced pulmonary vascular remodeling: a model for what human disease?," The Journal of clinical investigation, 106, pp. 733-738, 2000.

3. P. Davies, F. Maddalo, and L. Reid, "Effects of chronic hypoxia on structure and reactivity of rat lung microvessels," Journal of applied physiology: respiratory, environmental and exercise physiology, 58, pp. 795$801,1985$.

4. A. Hislop and L. Reid, "New findings in pulmonary arteries of rats with hypoxia-induced pulmonary hypertension," British journal of experimental pathology, 57, pp. 542-554, 1976.

5. R. J. van Suylen, J. F. Smits, and M. J. Daemen, "Pulmonary artery remodeling differs in hypoxia- and monocrotaline-induced pulmonary hypertension," American Journal of Respiratory and Critical Care Medicine : An Official Journal of the American Thoracic Society, Medical Section of the American Lung Association, 157, pp. 1423-1428, 1998.

6. D. J. Horstman, D. U. Frank, and G. F. Rich, "Prolonged inhaled NO attenuates hypoxic, but not monocrotalineinduced, pulmonary vascular remodeling in rats," Anesth Analg, 86, pp. 74-81, 1998.

7. T. D. Le Cras, D. H. Kim, S. Gebb, N. E. Markham, J. M. Shannon, R. M. Tuder, and S. H. Abman, "Abnormal lung growth and the development of pulmonary hypertension in the Fawn-Hooded rat," The American Journal of Physiology, 277, pp. L709-718, 1999.

8. K. Sato, S. Webb, A. Tucker, M. Rabinovitch, R. F. O'Brien, I. F. McMurtry, and T. J. Stelzner, "Factors influencing the idiopathic development of pulmonary hypertension in the fawn hooded rat," The American Review of Respiratory Disease, 145, pp. 793-797, 1992.

9. H. G. Zimmer, W. Zierhut, R. C. Seesko, and A. E. Varekamp, "Right heart catheterization in rats with pulmonary hypertension and right ventricular hypertrophy," Basic Res Cardiol, 83, pp. 48-57, 1988.

10. Y. Mitani, K. Maruyama, and M. Sakurai, "Prolonged administration of L-arginine ameliorates chronic pulmonary hypertension and pulmonary vascular remodeling in rats," Circulation, 96, pp. 689-697, 1997.

11. S. Singhal, R. Henderson, K. Horsfield, K. Harding, and G. Cumming, "Morphometry of the human pulmonary arterial tree," Circ Res, 33, pp. 190-197, 1973.

12. K. Horsfield, "Morphometry of the small pulmonary arteries in man," Circulation research, 42, pp. 593-597, 1978.

13. C. A. Dawson, G. S. Krenz, K. L. Karau, S. T. Haworth, C. C. Hanger, and J. H. Linehan, "Structure-function relationships in the pulmonary arterial tree," Journal of applied physiology: respiratory, environmental and exercise physiology, 86, pp. 569-583, 1999.

14. C. A. Dawson, G. S. Krenz, and J. H. Linehan, "Complexity and Structure-Function relationships in the pulmonary arterial tree," in Lung Biology in Health and Disease, Complexity in Structure and Function of the Lung, H. T. Robertson, Ed. New York: Marcel Dekker, inc., 1998, pp. 401-427.

15. Z. L. Jiang, G. S. Kassab, and Y. C. Fung, "Diameter-defined Strahler system and connectivity matrix of the pulmonary arterial tree," Journal of applied physiology: respiratory, environmental and exercise physiology, 76, pp. 882-892, 1994.

16. J. J. Fredberg and A. HOENIG, "Mechanical response of the lungs at high frequencies," Journal of Biomechanics Engineering, 100, pp. 57-66, 1978.

17. J. J. Fredberg, "Airway dynamics: recursiveness, randomness, and reciprocity in linear system simulation and parameter esti-mation.," in Lung Biology in Health and Disease, Respiratory Physiology: An Analytical Approach, M. Paiva, Ed., 40 ed. New York: Marcel Dekker, Inc., 1989, pp. 167-194.

18. K. L. Karau, R. C. Molthen, A. Dhyani, S. T. Haworth, C. C. Hanger, D. L. Roerig, R. H. Johnson, and C. A. Dawson, "Pulmonary arterial morphometry from microfocal X-ray computed tomography," Am J Physiol Heart Circ Physiol, 281, pp. H2747-2756, 2001.

19. L. A. Feldkamp, L. C. DAVIS, and J. W. KRESS, "Practical cone-beam algorithm," Journal of the Optical Society of America A, 1, pp. 612-619, 1984.

$20 . \quad$ K. L. Karau, R. H. Johnson, R. C. Molthen, A. H. Dhyani, S. T. Haworth, C. C. Hanger, D. L. Roerig, and C. A. Dawson, "Microfocal X-ray CT imaging and pulmonary arterial distensibility in excised rat lungs," Am J Physiol Heart Circ Physiol, 281, pp. H1447-1457, 2001. 
21. R. Molthen, Haworth, S., Heinrich, A., Dawson, C., "Measuring the Effect of Airway Pressure on Pulmonary Arterial Diameter," Medical Imaging: Physiology and Function - Methods, Systems, and Applications, A. A. A. a. A. V. Clough, 5031, pp. 195-203, SPIE, City, San Diego, CA, 2003.

22. R. Robb, D. Hanson, R. Karwoski, A. Larson, E. Workman, and M. Stacy, "Analyze: a comprehensive, operator-interactive software package for multidimensional medical image disply and analysis," Computerized Medical Imaging and Graphics, 13, pp. 433-454, 1989.

23. R. Robb, "The Biomedical Imaging Resource at Mayo Clinic Guest Editorial," IEEE Transactions on Medical Imaging, 20, pp. 854-867, 2001.

24. $\quad$ Y. C. Fung, Biomechanics: circulation, Springer, New York, 1997.

25. A. King, P. Smith, and D. Heath, "Ultrastructural differences between pulmonary arteriolar muscularization induced by hypoxia and monocrotaline," Exp Mol Pathol, 61, pp. 24-35, 1994.

26. R. Mathew, E. S. Gloster, T. Sundararajan, C. I. Thompson, G. A. Zeballos, and M. H. Gewitz, "Role of inhibition of nitric oxide production in monocrotaline-induced pulmonary hypertension," J Appl Physiol, 82, pp. 1493-1498, 1997. 